\title{
Heterogenität der Beschäftigungsdynamik und Segmentierungsphänomene auf dem deutschen Arbeitsmarkt
}

\author{
Bernhard Boockmann • Susanne Steffes
}

Angenommen: 25. Januar 2011 / Online publiziert: 9. Februar 2011

(C) Institut für Arbeitsmarkt- und Berufsforschung 2011

Zusammenfassung Der Artikel präsentiert empirische Ergebnisse und Schlussfolgerungen zur Frage der betriebliche Mobilität von Arbeitnehmern und ihrer Determinanten vor dem Hintergrund verschiedener ökonomischer Theorien und unter Berücksichtigung der Institutionen des deutschen Arbeitsmarktes. Ziel ist es insbesondere, diejenigen Teilbereiche des Arbeitsmarktes zu identifizieren, die besonders dynamisch oder die besonders inflexibel sind. Schlussfolgerungen betreffen einerseits die Personalpolitik der Betriebe, andererseits gezielte Förderungen zur Integration von Arbeitslosen in stabile Erwerbsverhältnisse.

\section{Heterogeneous employment dynamics and segmentation on the German labor market}

\begin{abstract}
This paper presents recent empirical evidence on the extent and determinants of employment dynamics and job mobility, building on economic theory and German labor market institutions. In particular, it identifies dynamic and immobile segments of the labor market. Conclusions are drawn with respect to companies' personnel policies, as well
\end{abstract}

B. Boockmann ( $₫)$

Institut für Angewandte Wirtschaftsforschung,

Ob dem Himmelreich 1, 72074 Tübingen, Deutschland

e-mail: bernhard.boockmann@iaw.edu

B. Boockmann

Universität Tübingen, Tübingen, Deutschland

B. Boockmann

IZA, Bonn, Deutschland

S. Steffes

Zentrum für Europäische Wirtschaftsforschung, Mannheim,

Deutschland as to public policy aimed at integrating the unemployed into stable employment relationships.

\section{Projektziele}

Als Grund für die hohe und persistente Arbeitslosigkeit in Deutschland während der letzten beiden Jahrzehnte wird in der öffentlichen Diskussion immer wieder die Inflexibilität des deutschen Arbeitsmarktes angeführt. Die Ausgestaltung des Kündigungsschutzes, der Einfluss von Betriebsräten auf die Personalpolitik und insbesondere personelle $\mathrm{Zu}$ - und Abgänge, aber auch betriebliche Institutionen und Vorgehensweisen wie die Senioritätsentlohnung werden als Ursachen dafür herangezogen, dass die Mobilität der Arbeitnehmer über Betriebe sowie über Branchen, Berufe und Regionen im internationalen Vergleich relativ gering ist, was zu einem unzureichendem Ausgleich am Arbeitsmarkt beiträgt. Im Gegensatz dazu ist in der öffentlichen Diskussion die Einschätzung, dass sich die Risiken für die Arbeitnehmer erhöht haben und traditionelle Muster wie das „Lebensarbeitsverhältnis“ an Bedeutung verlieren, weit verbreitet.

Zum Startzeitpunkt des DFG-Schwerpunktprogramms „Flexibilisierungspotenziale bei heterogenen Arbeitsmärkten" war es aus wissenschaftlicher Sicht nicht ausreichend empirisch untersucht, in welchen Segmenten der Arbeitsmarkt besonders hohe Inflexibilitäten aufweist und welchen Einfluss Institutionen, Gesetzgebung und unterschiedliche individuelle und betriebliche Ausgangslagen darauf haben. Im Projekt „Beschäftigungsdynamik und heterogene Arbeitsverhältnisse: Untersuchungen zur Arbeitsmarktsegmentierung auf betrieblicher Ebene" wurde deshalb die betriebliche Mobilität von Arbeitnehmern und ihre Determinanten vor dem Hintergrund verschiedener ökonomischer Theorien und unter Berücksichtigung der Institutionen des 
deutschen Arbeitsmarktes empirisch untersucht. Ziel war es insbesondere, diejenigen Teilbereiche des Arbeitsmarktes zu identifizieren, die besonders dynamisch oder die besonders inflexibel sind.

Grundlegend für die Analyse ist die gleichzeitige Betrachtung der Betriebs- und der Arbeitnehmerseite. Die zeitliche Stabilität oder Instabilität der Beschäftigungsverhältnisse kann zunächst auf die Bedarfe der Arbeitgeber zurückzuführen sein. Einerseits müssen Betriebe Schwankungen der Geschäftstätigkeit bewältigen und ihre Beschäftigung daran anpassen. Andererseits haben Arbeitgeber insbesondere dann ein Interesse an stabilen Beschäftigungsverhältnissen, wenn Arbeitnehmer besondere Fähigkeiten aufweisen und es schwierig und mit hohen Kosten verbunden wäre, neue Arbeitskräfte zu finden. Stabilität oder Instabilität können aber ebenso vom Arbeitnehmer ausgehen und durch den Wunsch nach Vermeidung von Risiken einerseits sowie durch das Bestreben, sich beruflich weiterzuentwickeln, anderseits hervorgerufen werden. Diese Einflüsse interagieren miteinander. Im günstigen Fall finden sich Zonen der Mobilität und der Stabilität als Ergebnis eines freiwilligen Sortierprozesses, wonach Arbeitnehmer und Arbeitgeber mit gleich gelagerten Interessen auf dem Arbeitsmarkt zusammenkommen. Im ungünstigen Fall entstehen diese unterschiedlichen Zonen als Ergebnis eines Segmentierungsprozesses, auf dem bestimmte Arbeitnehmer unfreiwillig über längere Zeit in instabilen Beschäftigungsverhältnissen verbleiben.

Die Beschäftigungsdynamik wird in den im Folgenden dargestellten Ergebnissen auf der individuellen und der betrieblichen Ebene gemessen. Im ersten Fall werden Beschäftigungsdauern und Abgangswahrscheinlichkeiten auf der individuellen Ebene (Arbeitnehmer) und deren Determinanten analysiert. Im zweiten Fall werden die Fluktuationsraten auf der Betriebsebene untersucht und insbesondere auf konjunkturelle Schwankungen zurückgeführt.

Unter den Institutionen werden vor allem der gesetzliche Kündigungsschutz und die Betriebsverfassung betrachtet. Eine vertiefende Analyse wird zudem für die Beschäftigungsdynamik von Frauen nach der Geburt des ersten Kindes vorgenommen. Vor dem Hintergrund unterschiedlicher Erwerbskarrieren von Frauen und Männern mit unterschiedlich langen Kindererziehungszeiten ist dies ein Aspekt, der in Bezug auf den Fachkräftemangel immer wichtiger wird.

Als Datengrundlage für fast alle Forschungsarbeiten dieses Projektes dienen die verknüpften Personen- und Betriebsdaten des Instituts für Arbeitsmarkt- und Berufsforschung an der Bundesagentur für Arbeit (Linked EmployerEmployee-Datensatz des IAB, kurz LIAB), die erst seit Beginn des DFG-Schwerpunktprogramms für die Analyse zur Verfügung stehen. Sie basieren auf einer jährlichen Befragung von Betrieben (Betriebspanel), der Angaben über die in diesen Betrieben Beschäftigten aus den Meldungen an die
Sozialversicherung (Beschäftigtenstatistik) zugespielt werden. Da sich der Familienkontext und die Personalpolitik der Unternehmen in diesen Daten nur unzureichend abbilden, wird für die Analyse der Beschäftigung von Frauen nach der Geburt eines Kindes ein Datensatz mit Personaldaten eines großen deutschen Unternehmens der Finanzdienstleistungsbranche verwendet. Aus den Originaldaten werden Karriereverläufe aller jungen Mütter, die im Zeitraum 2000-2004 ihr erstes Kind bekommen haben, erstellt. Für Vergleichsrechnungen liegen außerdem Erwerbsbiografiedaten von NichtMüttern desselben Unternehmens vor.

\section{Determinanten von Beschäftigungsdauern}

Mit den Daten des LIAB kann die Beschäftigungsdauer weitaus präziser nachverfolgt werden, als dies in der Vergangenheit möglich war (die folgenden Ergebnisse basieren auf Boockmann und Steffes 2010). Abbildung 1 zeigt, dass über 50 Prozent der im Zeitraum 1996 bis 2001 neu begonnenen Beschäftigungsverhältnisse nach zwei Jahren bereits wieder beendet waren. Dagegen dauert ein Viertel mehr als sechs Jahre. Auf dem deutschen Arbeitsmarkt treten also stabile und instabile Beschäftigungsverhältnisse gleichzeitig auf. Ein wichtiges weiteres Differenzierungsmerkmal neben der Beschäftigungsdauer ist der Folgezustand nach dem Ende der Beschäftigung: Handelt es sich um einen Übergang in Arbeitslosigkeit oder einen Arbeitgeberwechsel? Nur im ersten Fall würde man auf mögliche Instabilität in der Erwerbsbiographie schließen.

Für unterschiedliche Beschäftigungsdauern gibt es eine Vielzahl von Ursachen. Die Wahrscheinlichkeit, dem instabilen Segment anzugehören, ist beispielsweise für Arbeiter mit niedriger beruflicher Qualifikation höher als für Angestellte oder Arbeiter mit hoher Qualifikation. Dies gilt jedoch nur für Übergänge in Arbeitslosigkeit. Übergänge zu einem neuen Arbeitgeber weisen vor allem die Gruppen auf, für die Mobilität mit der Suche nach einem besser geeigneten Arbeitsverhältnis und Karrieremöglichkeiten verbunden ist. Akademiker haben zum Beispiel eine geringere Wahrscheinlichkeit, in Arbeitslosigkeit zu wechseln, weisen aber eine höhere Wahrscheinlichkeit des direkten Arbeitgeberwechsels auf.

Auch junge Arbeitnehmer wechseln häufiger den Arbeitgeber. Darüber hinaus zeigt sich eine überraschende Persistenz von Beschäftigungsverläufen. Arbeitnehmer, die vor der Aufnahme einer Beschäftigung arbeitslos waren, haben eine um (je nach Spezifikation und Bundesgebiet Ost und West) zwischen 260 und 310 Prozent höhere Wahrscheinlichkeit, wieder in Arbeitslosigkeit zu wechseln, als Arbeitnehmer, die zuvor beschäftigt waren.

Neben individuellen Eigenschaften gibt es eine ganze Reihe betrieblicher Eigenschaften, die einen Einfluss darauf haben, ob ein Beschäftigungsverhältnis stabil oder eher 
West

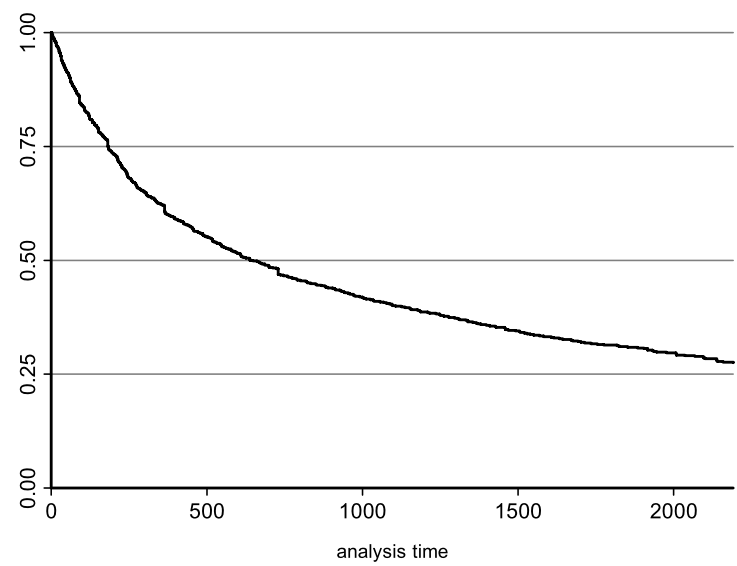

Ost

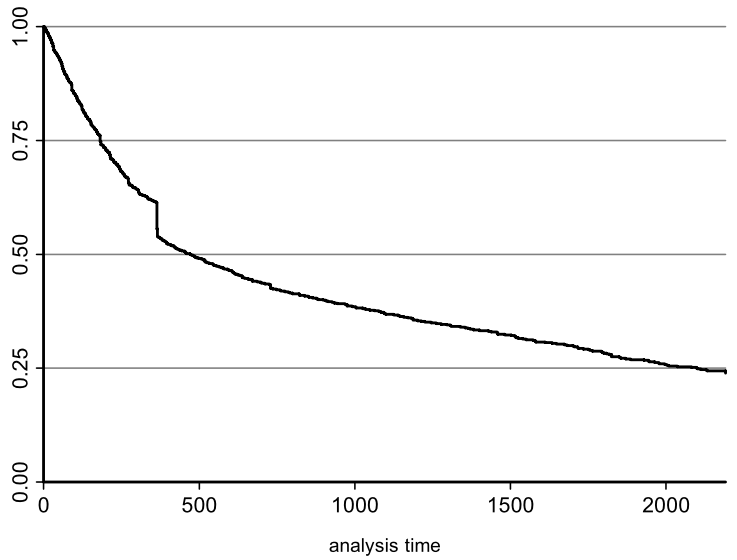

Anmerkung: Dargestellt sind Kaplan-Meier-Überlebenswahrscheinlichkeiten für zwischen 1996 und 2001

begonnene Beschäftigungsverhältnisse in Abhängigkeit von der Dauer des Beschäftigungsverhältnisses.

Lesebeispiel: In Westdeutschland ist nach ca. 700 Tagen die Hälfte der begonnenen Beschäftigungsverhältnisse

wieder beendet. Quelle: Boockmann und Steffes (2010).

Abb. 1 Betriebliche Verweildauern in West- und Ostdeutschland

instabil ist. Deutlich wird vor allem die Rolle des Betriebsrates. Wo ein Betriebsrat vorhanden ist, vermindert sich die Wahrscheinlichkeit eines Abgangs in Arbeitslosigkeit ebenso wie die Wahrscheinlichkeit eines Arbeitgeberwechsels um jeweils etwa 20 bis 30 Prozent. Beides ist gut zu erklären: Einerseits haben Betriebsräte ein Informations- und Beratungsrecht bei Entlassungen, was sich negativ auf die Rate der Entlassungen auswirken kann. Andererseits haben sie eine Vermittlungsfunktion zwischen Arbeitnehmern und Unternehmensführung, was sich dämpfend auf das Klima und somit positiv auf die freiwilligen Kündigungen auswirken kann. Dieser Zusammenhang lässt sich allerdings nur für Arbeiter und nicht für Angestellte feststellen. Offenbar unterscheidet sich die Wirkung der Betriebsratstätigkeit nach diesen Zielgruppen.

Ein weiterer Befund ist, dass Betriebe, die in Weiterbildung und in Informations- und Kommunikationstechnologien investieren und damit die Produktivität und Kommunikationsfähigkeit ihrer Mitarbeiter erhöhen, eine höhere Stabilität der Beschäftigung aufweisen. Auch hier zeigt sich, dass die positiven Effekte der Weiterbildung nur für Beschäftigte, die einen Lehrberuf gelernt haben, und nicht für Ungelernte und Akademiker gelten.

Aus den Ergebnissen lässt sich auch folgern, dass Arbeitnehmer mit Eigenschaften, die sich negativ auf die Stabilität auswirken, eher in Betrieben arbeiten, die generell eine geringere Stabilität der Beschäftigung aufweisen. Dies gilt beispielsweise für zuvor arbeitslos gewesene Beschäftigte. Unterschiede zu vorher beschäftigten Arbeitnehmern lassen sich nicht ausschließlich darauf zurückführen, dass Betriebe ehemalige Arbeitslose eher wieder entlassen, sondern auch darauf, dass ehemalige Arbeitslose gezwungen sind, eine Beschäftigung bei Betrieben mit höherer Fluktuation anzunehmen.

Eine Frage, die sich in der öffentlichen Diskussion um Flexibilität am Arbeitsmarkt immer wieder stellt, ist die nach der Wirkung von Kündigungsschutzregelungen. Empirisch ist es nicht einfach, kausale Effekte solcher Regelungen zu untersuchen, weshalb man sich oft Gesetzesänderungen zunutze macht. In Boockmann et al. (2008) wird die Tatsache, dass sich der Schwellenwert für Betriebe, die unter das Kündigungsschutzgesetz fallen, im Jahr 1999 geändert hat, dazu genutzt, um die Wirkung auf die Stabilität neuer Beschäftigungsverhältnisse zu untersuchen. Die Frage ist insofern interessant, als bisherige Studien teilweise keinen Effekt des Kündigungsschutzes auf die Abgänge von Beschäftigten aus den Betrieben festgestellt haben (Bauer et al. 2007). Diese Studien stützen insofern das theoretische Argument von Lazear (1990), wonach der Kündigungsschutz so kompensiert werden kann, dass er unwirksam wird.

Die Ergebnisse zeigen, dass sich der Kündigungsschutz signifikant positiv auf die Dauer des Arbeitsverhältnisses auswirkt. Die Effekte sind in der Größenordnung beträchtlich: Sie erhöhen die Wahrscheinlichkeit, ein Jahr nach der Aufnahme einer Beschäftigung noch bei demselben Betrieb beschäftigt zu sein, um 10 Prozentpunkte Dies gilt erst nach etwa 200 Tagen, was mit dem Einsetzen des Kündigungsschutzes im Anschluss an die übliche Probezeit von sechs Monaten übereinstimmt. Allerdings ist darauf hinzuweisen, dass die Ergebnisse nicht ohne weiteres von den betrachteten Kleinbetrieben auf größere Betriebe übertragen werden können. 


\section{Stabilität über die Dauer der Beschäftigung}

Nach unterschiedlichen arbeitsmarktökonomischen Theorien kann sich die Stabilität von Beschäftigungsverhältnissen im Zeitablauf aus verschiedenen Gründen erhöhen (Boockmann und Hagen 2008). Erstens können von vorneherein auf kurze Dauer angelegte Beschäftigungsverhältnisse (zum Beispiel bei befristeten Tätigkeiten) fortfallen, wodurch die verbleibenden Beschäftigungsverhältnisse im Durchschnitt stabiler werden. Zweitens werden Beschäftigungsverhältnisse, bei denen Arbeitnehmer und Stelle schlecht zusammenpassen, im Laufe der Zeit sukzessive beendet, wenn sich der geringe Grad der Übereinstimmung herausstellt. Drittens erwerben die Arbeitnehmer im Lauf der Zeit betriebsspezifisches Wissen, was die Kündigungswahrscheinlichkeit auf Arbeitnehmer- und auf Arbeitgeberseite verringert.

Durch diese Mechanismen wandelt sich auch der Einfluss individueller und betrieblicher Ausgangsbedingungen auf die Abgangswahrscheinlichkeit mit der Fortdauer des Beschäftigungsverhältnisses. Empirisch war bisher unklar, ob der Einfluss oben genannter individueller, betrieblicher und institutioneller Eigenschaften mit der Dauer der Beschäftigung ansteigt, konstant bleibt oder sinkt. In Boockmann und Steffes (2007) wird deshalb die Veränderung der Determinanten über die Dauer hinweg für in den Jahren 1996 und 1997 neu gegründete Beschäftigungsverhältnisse untersucht. Die Größe der betriebsspezifischen Einflüsse verändert sich zum Teil erheblich über die Dauer des Beschäftigungsverhältnisses. So steigt die Größe der Koeffizienten für den Betriebsrat und die Investitionen in Informationsund Kommunikationstechnologie über die Dauer deutlich an. Hinsichtlich des Betriebsrates bedeutet das, dass die Stabilisierung der Beschäftigungsverhältnisse durch den Betriebsrat den Langzeitbeschäftigten in wesentlich höherem Maße zugute kommt als Beschäftigten, die weniger lange im Betrieb beschäftigt sind. Insofern verstärkt der Betriebsrat die Teilung des Arbeitsmarktes in bewegliche und stabile Segmente.

\section{Beschäftigungsanpassungen im Konjunkturverlauf}

In einem vollkommen flexiblen Arbeitsmarkt ist gewährleistet, dass Betriebe sich an Nachfrageschwankungen (etwa konjunkturelle Schwankungen) anpassen und die Beschäftigung verändern können. Neben Kündigungen aus diesen „betriebsbedingten“ Gründen unterliegen auch freiwillige Kündigungen seitens der Arbeitnehmer konjunkturellen Schwankungen. Bei steigender Arbeitsnachfrage und damit einer steigenden Anzahl an Alternativen mit höheren Lohnangeboten steigt auch die Zahl der Arbeitgeberwechsel (Bachmann 2005). Vermutlich gilt das vor allem für die Arbeitnehmer, die aufgrund von kurzen Beschäftigungsverhältnissen noch nicht sehr viel betriebsspezifisches Wissen angehäuft haben. In diesem Bereich sind stärker ausgeprägte konjunkturelle Einflüsse zu erwarten.

In diese Anpassungsmechanismen greift die deutsche Gesetzgebung dadurch ein, dass Kündigungsschutzregelungen und weitere Regelungen die Beschäftigung gegenüber negativen Nachfrageschwankungen stabilisieren. Bisher liegen nur für andere Länder Studien vor, die den Einfluss von Nachfrageschocks auf die Beschäftigungsschwankungen heterogener Arbeitnehmergruppen empirisch untersuchen (siehe zum Beispiel Gielen und van Ours 2006). Ferner wurde nicht analysiert, ob bei den Anpassungsvorgängen hinsichtlich der Betriebszugehörigkeit Unterschiede bestehen. Um diese Zusammenhänge empirisch für Deutschland zu überprüfen, werden die Effekte von Nachfrageschwankungen auf den betrieblichen Beschäftigungsauf- und -abbau sowie auf betriebliche Einstellungs- und Entlassungsraten für den Zeitraum 1996 bis 2005 analysiert (Steffes 2010). Dabei wird zwischen jährlichen betriebs- und branchenspezifischen bzw. gesamtwirtschaftlichen Schwankungen in der Wertschöpfung bzw. den Umsätzen unterschieden.

Zur Überprüfung der Hypothesen wird zwischen Abgängen nach kurzer, mittlerer und langer Beschäftigungsdauer sowie nach Übergängen in Arbeitslosigkeit oder zu einem neuen Arbeitgeber unterschieden. Die Unterscheidung zwischen Folgezuständen approximiert die Unterscheidung zwischen arbeitgeber- und arbeitnehmerseitigen Kündigungen, da Entlassungen seitens des Arbeitgebers zu einem großen Anteil in Arbeitslosigkeit und freiwillige Kündigungen überwiegend in einem neuen Beschäftigungsverhältnis münden.

Die Ergebnisse zeigen, dass die Auswirkungen von betriebsspezifischen Schwankungen auf die Beschäftigung in der Größenordnung gering sind, wofür es zwei Gründe geben kann. Einerseits könnten diese Schwankungen eher von kurzfristiger Natur sein und die Betriebe könnten diese dementsprechend gut kompensieren. Andererseits könnte es aber auch bedeuten, dass diese Art der Schwankungen durch andere Maßnahmen wie zum Beispiel Lohn- oder Arbeitszeitanpassungen ausgeglichen wird. Die Effekte von branchenspezifischen bzw. gesamtwirtschaftlichen Schwankungen sind wesentlich höher, treten aber nicht in allen Branchen und Betrieben gleichermaßen auf. Die Unterscheidung nach kurzen, mittleren und langen Beschäftigungsdauern zeigt, dass vor allem die Beendigung kurz andauernder Beschäftigungsverhältnisse auf diese Schwankungen reagiert. Dies betrifft sowohl Abgänge in Arbeitslosigkeit als auch Übergänge zu einem neuen Arbeitgeber. Dies bestätigt die Hypothese, dass kurze Beschäftigungsverhältnisse zu einem höheren Maße konjunkturabhängig sind als lange Beschäftigungsverhältnisse. Weiterhin zeigt sich, dass die Stabilität der Beschäftigung eher von den Eigenschaften der Betriebe und der Arbeitnehmer als von Nachfrageschwankungen abhängt. 


\section{Beschäftigungsstabilität nach einer Geburt}

Eine wichtige Komponente zur Sicherung des Fachkräftebestandes und damit der Beschäftigungsstabilität aus betrieblicher Sicht ist die Nutzung des Arbeitsmarktpotenzials von Frauen. Heutzutage sind Frauen hervorragend ausgebildet und erobern zunehmend von Männern dominierte Berufsfelder und Positionen. Dennoch gilt nach wie vor, dass vorwiegend Frauen Verantwortung für die Kindererziehung übernehmen und ihre Karriere nach der Geburt eines Kindes oftmals für längere Zeit unterbrechen. Dies führt zu ökonomischen Verlusten für die Frauen selbst und für die Unternehmen, in denen diese Frauen beschäftigt sind. Aus einer ökonomischen Perspektive sind deshalb kürzere Elternzeitdauern und hohe Rückkehrraten eine wichtige Zielvariable. Dies gilt vor allem für diejenigen Frauen, die vor der Geburt des ersten Kindes im Unternehmen Karriere gemacht und demnach viel betriebsspezifisches Wissen akkumuliert haben.

Bisherige Studien haben für Deutschland das Rückkehrverhalten von Müttern nach der Geburt eines Kindes im Zusammenhang mit Änderungen in der Gesetzgebung (Ondrich et al. 1996; Schönberg und Ludsteck 2003) oder unter Berücksichtigung familiärer Hintergründe (Geyer und Steiner 2007; Kuhlenkasper und Kauermann 2009) untersucht. Die Dauer der Elternzeit und die Rückkehrwahrscheinlichkeit in das frühere Unternehmen hängt neben persönlichen und gesetzgeberischen Umständen jedoch auch stark von den Möglichkeiten ab, die das Unternehmen zur Vereinbarkeit von Familie und Beruf bietet. Darüber gibt es in repräsentativen Datensätzen in der Regel keine ausreichenden Informationen. Daher wurden in Fitzenberger et al. (2010)
Personaldaten eines Unternehmens der Finanzbranche verwendet, was den Vorteil hat, dass alle Frauen derselben Personalpolitik unterliegen. Die vorliegende Studie ist darüber hinaus die erste, in welcher der Zusammenhang zwischen einem erfolgreichen Karriereverlauf vor der Geburt des ersten Kindes und dem Rückkehrverhalten nach der Geburt untersucht wird.

Folgende Zielzustände für das Ende der Elternzeit werden unterschieden: Rückkehr in das Unternehmen, Geburt eines zweiten Kindes und Austritt aus dem Unternehmen. Außerdem wurde eine Kategorie für Teilzeit in Elternzeit gebildet, an die sich noch einmal die drei vorgenannten Zielzustände anschließen können. Als positive Zielgröße aus Sicht des Unternehmens wurde die Rückkehr in das Unternehmen (innerhalb von vier Jahren) und die Stabilität der Beschäftigung für mindestens ein Jahr nach der Rückkehr definiert. Als negative Zielgröße wurde die Beendigung des Beschäftigungsverhältnisses definiert. Wie die Geburt eines zweiten Kindes während der ersten Elternzeit zu bewerten ist, bleibt unklar. Auf der einen Seite wird dadurch die Dauer der Unterbrechung verlängert, auf der anderen Seite besteht weiterhin die Möglichkeit der Rückkehr in das Unternehmen.

Wie in Abb. 2 zu sehen ist, kehren nur etwa 50 Prozent aller Mütter innerhalb der ersten vier Jahre in das Unternehmen zurück, nur 20 Prozent in Vollzeitjobs. Von den 50 Prozent der Rückkehrenden verlassen noch einmal etwa 10 Prozent im ersten Jahr nach ihrer Rückkehr das Unternehmen.

In den Regressionsanalysen wurden diese Befunde weiter differenziert. Dazu wurden Maße für den bisherigen Karriereverlauf einer Arbeitnehmerin als unabhängige Variablen
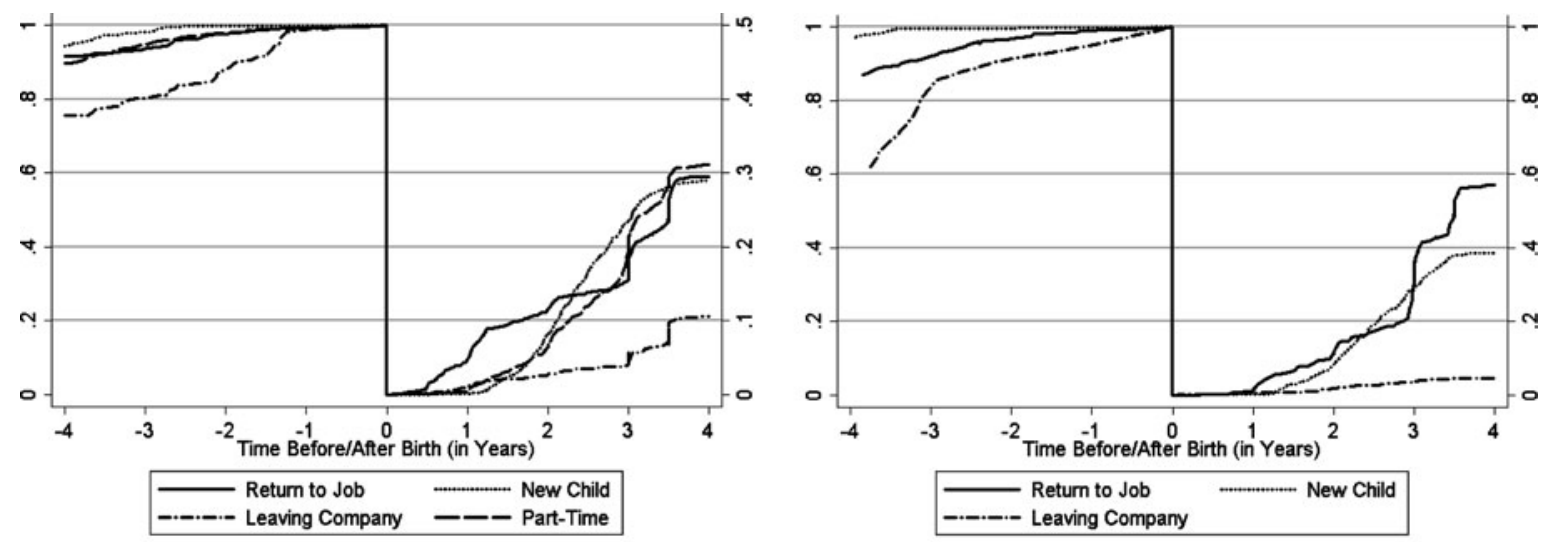

Anmerkung: Die Abbildung zeigt - jeweils für die vier Zielzustände - den Anteil der Mütter, die vor der Geburt ( -4 bis 0 Jahre) bereits im Unternehmen waren und die nach der Geburt ( 0 bis 4 Jahre) in den jeweiligen Zustand gewechselt sind; linke Grafik: alle direkten Übergänge einschließlich Teilzeit in Elternzeit; rechte Grafik: alle Übergänge nach Teilzeit in Elternzeit. Quelle: Fitzenberger et al. (2010).

Abb. 2 Rückkehr von Frauen nach dem Erziehungsurlaub in den Betrieb 
herangezogen. Folgende Maße wurden dazu definiert: Beförderung durch Aufstieg in der Lohngruppe, Leitungsfunktion und relative Lohnposition. Letzteres ist ein Maß, das auch die Vergleichsgruppe der Nicht-Mütter mit einbezieht und somit ihre Leistung im Vergleich der Gruppe, die der Mutter am ähnlichsten ist, berücksichtigt.

In den Analysen zeigt sich, dass die Beschäftigungsstabilität nach der Geburt eines Kindes für Frauen mit Leitungsfunktionen und hohen relativen Lohnpositionen höher ist als für andere Frauen. Obwohl die Geburt eines Kindes dramatische Einschnitte in den Erwerbsverlauf von Frauen in diesem Unternehmen nach sich zieht, gelingt es offensichtlich eher, solche Frauen an das Unternehmen zu binden, die bereits einen erfolgreichen Karriereweg eingeschlagen haben.

\section{Schlussfolgerungen und Diskussion}

Die Ergebnisse dieses Teilprojektes geben einen vertieften Einblick in die Dynamik des deutschen Arbeitsmarktes. Hinsichtlich der individuellen und betrieblichen Merkmale lassen sich Zonen der Mobilität und Stabilität voneinander abgrenzen. Im Folgenden werden die wirtschaftspolitischen und personalwirtschaftlichen Schlussfolgerungen aus den Befunden zusammengefasst.

Für die Betriebe ist die Schlussfolgerung zu ziehen, dass durch bestimmte betriebliche Strategien die Beschäftigungsstabilität gezielt erhöht werden kann. Im Wettbewerb um Fachkräfte müssen Betriebe gegebenenfalls neue und innovative Wege gehen, um die Attraktivität für ihr Personal zu steigern und die Mitarbeiterbindung zu erhöhen. Die Analysen zeigen zum Beispiel, dass Weiterbildungsangebote wie auch die Einführung moderner Kommunikationstechnologien mit einer höheren Mitarbeiterbindung zusammenhängen. Zukünftige Forschung sollte sich verstärkt mit den personalpolitischen Möglichkeiten der Mitarbeiterbindung befassen, da dies von zentraler Bedeutung für das Human Ressource Management von Unternehmen ist. Die LIAB-Daten bieten hierfür hervorragende Möglichkeiten, da nicht nur einzelne Maßnahmen sondern ganze Bündel von Maßnahmen bzw. personalpolitischen Strategien untersucht werden können. Weiterhin könnte mit diesen Daten analysiert werden, welche Betriebe mit welchen Maßnahmen erfolgreich bei der Bindung der „besten Köpfe“ sind. Angesichts eines immer wieder konstatierten Fachkräftemangels ist dies im Wettbewerb der Unternehmen von höchster Relevanz.

Attraktive Angebote sind vor allem für Frauen, die zwischen Karriere und Familie stehen, notwendig. Nicht nur gesellschaftliche Überlegungen sondern auch die Notwendigkeit, nicht genutzte Potenziale für die Kompensation des Fachkräftemangels zu aktivieren, spricht für eine bessere Vereinbarkeit von Familie und Beruf in den Unternehmen. Dies muss von Politik und Gesellschaft unterstützt werden, liegt aber auch in der Verantwortung der einzelnen Unternehmen. Ein langsamer Wiedereinstieg, wie ihn die Teilzeit in Elternzeit ermöglicht, sowie flexible Arbeitszeitmodelle (80 Prozent der Rückkehrerinnen arbeiten Teilzeit) könnten solche Möglichkeiten bieten. Bei der Evaluierung von Angeboten speziell für Eltern steckt die empirische Forschung noch in den Kinderschuhen. Auch hier gibt es Möglichkeiten, mit den LIAB-Daten zu arbeiten, aber auch Einzelunternehmensdaten bieten gute Chancen, wenn es „natürliche Experimente" für eine Evaluation gibt.

In wirtschaftspolitischer Hinsicht sind vor allem Tendenzen zu einer Segmentierung des Arbeitsmarktes zu bedenken, die sich aus den empirischen Befunden interpretieren lassen. Vor allem Arbeitnehmer mit Arbeitslosigkeitserfahrung sind von einer Aufeinanderfolge von kurzfristigen Beschäftigungsverhältnissen und weiterer Arbeitslosigkeit betroffen, die es erschwert, die eigene Erwerbsbiographie zu stabilisieren. Betriebsräte wirken dem nicht ausreichend entgegen, vielmehr schützen sie vor allem die längerfristig Beschäftigten vor dem Arbeitsplatzverlust, was die Segmentierungstendenzen eher verstärkt als mindert. Zudem konzentriert sich die Kurzfristbeschäftigung in bestimmten Betrieben. Schließlich wird deutlich, dass die „Kurzfristbeschäftigten“ auch von konjunkturellen Schwächephasen stärker betroffen sind als andere Arbeitnehmer.

Neben dieser gesamtwirtschaftlich unerwünschten Segmentierung darf andererseits nicht übersehen werden, dass ein Teil der festgestellten Unterschiede die beiderseitigen Interessen widerspiegeln und eine Aufteilung in instabile und stabile Teilbereiche des Arbeitsmarktes zumindest teilweise von beiden Seiten freiwillig ist. Gerade jüngere und qualifizierte Arbeitnehmer wechseln den Arbeitgeber häufig, um sich beruflich zu verbessern. Sinnvoller als die Änderung pauschaler gesetzlicher Regelungen, die für alle Arbeitnehmer Anwendung finden, ist es daher, die Integration von Arbeitslosen in den Betrieben zu verbessern. Hierzu sollten die vorhandenen Möglichkeiten zur Qualifikation und Weiterbildung verbessert und vermehrt öffentliche Förderungen bereitgestellt werden. Aufgrund einer Beschränkung der Datenbasis bis 2002 am Anfang des Projektes und einer Erweiterung bis 2006 zu einem späteren Zeitpunkt, konnte eine Analyse der Hartz-Reformen (Agenda 2010) nicht durchgeführt werden. Zukünftig sollte dies jedoch nachgeholt werden. Vor allem die Flexibilisierungsmaßnahmen im Hinblick auf Leih- und Zeitarbeit sowie befristeter Beschäftigung hatte zum Ziel, die Segmentierung des Arbeitsmarktes aufzulockern. Es stellt sich nun die empirisch zu beantwortende - Frage, inwieweit dies gelungen ist.

Darüber hinaus wäre es interessant zu analysieren, warum der deutsche Arbeitsmarkt relativ gut über die vergangene Wirtschafts- und Finanzkrise hinweggekommen ist. Aktuelle Zahlen beziffern die Arbeitslosenquote auf einem Tief- 
stand seit zwei Jahrzehnten. Als Gründe werden hierfür immer wieder die ausgebaute Kurzarbeit wie auch die Agenda 2010 gesehen. Kausale Evidenz gibt es hierfür jedoch bisher nicht. Die in Abschn. 4 vorgestellte Analyse hat gezeigt, dass konjunkturelle Schwankungen im Beobachtungszeitraum einen relativ kleinen Anteil an der gesamten Arbeitsmarktdynamik ausgemacht haben. Wenn man nur die neunziger Jahre betrachtet, gibt es sogar einen positiven Zusammenhang zwischen BIP und Übergängen in Arbeitslosigkeit. Es könnte also sein, dass viele deutsche Betriebe Ende der neunziger Jahre ihre Belegschaften reduziert und dadurch ihre Kosteneffizienz und Wettbewerbsfähigkeit gestärkt haben.

\section{Literatur}

Bachmann, R.: Labour market dynamics in Germany: hirings, separations, and job-to-job transitions over the business cycle. SFB 649 discussion papers 2005-045, Sonderforschungsbereich 649, Humboldt University, Berlin (2005)

Bauer, T.K., Bender, S., Bonin, H.: Dismissal protection and worker flows in small establishments. Economica 74, 804-821 (2007)

Boockmann, B., Steffes, S.: Seniority and job stability: a quantile regression approach using matched employer employee data. ZEW discussion paper No. 07-014 (2007)

Boockmann, B., Steffes, S.: Workers, firms or institutions: what determines job duration for male employees in Germany? Ind. Labor Relat. Rev. 64(2010), 853-871 (2010)

Boockmann, B., Hagen, T.: Fixed-term contracts as sorting mechanisms: evidence from job durations in west Germany. Labour Econ. 15(2008), 984-1005 (2008)

Boockmann, B., Gutknecht, D., Steffes, S.: Die Wirkung des Kündigungsschutzes auf die Stabilität junger Beschäftigungsverhältnisse. Z. ArbeitsmarktForsch. 41, 347-364 (2008)

Fitzenberger, B., Steffes, S., Strittmatter, A.: Individual performance and employment behavior after maternity leave: evidence based on company data. Manuskript, Universität Freiburg und ZEW (2010)

Geyer, J., Steiner, V.: Short-run and long-run effects of childbirth on mothers' employment and working hours across institutional regimes: an empirical analysis based on the European community houshold panel. IZA discussion paper Nr. 2693 (2007)
Gielen, A., van Ours, J.: Age-specific cyclical effects in job reallocation and labor mobility. Labour Econ. 13(4), 439-504 (2006)

Kuhlenkasper, T., Kauermann, G.: Duration of maternity leave in Germany: a case study of nonparametric hazard models and penalized splines. SOEP papers, Nr. 213, DIW Berlin (2009)

Lazear, E.P.: Job security provisions and employment. Q. J. Econ. 105(3), 699-726 (1990)

Ondrich, J., Spiess, K.C., Yang, Q.: Barefoot and in a German kitchen: federal parental leave and benefit policy and the return to work after childbirth in Germany. J. Popul. Econ. 9(3), 247-66 (1996)

Schönberg, U., Ludsteck, J.: Maternity leave legislation, female labor supply, and the family wage gap. IZA discussion paper No. 2699 (2007)

Steffes, S.: Employment dynamics, cyclical shocks, and heterogeneity: an empirical analysis with firm-level data. Manuskript, ZEW (2010)

Bernhard Boockmann ist seit dem 1. November 2007 wissenschaftlicher Geschäftsführer des Instituts für Angewandte Wirtschaftsforschung (IAW), Tübingen. Er studierte Volkswirtschaftslehre und Philosophie in Heidelberg und Mannheim und Volkswirtschaftslehre am University College London und schloss seine Promotion 1998 an der Universität Mannheim ab. In seinen wissenschaftlichen Arbeiten befasst er sich mit Arbeitsmarkpolitik, individuellen Erwerbsverläufen, der Wirkung von Arbeitsmarktregulierungen und der Lohnbildung. Weitere Arbeitsgebiete sind die ökonomische Analyse politischer Entscheidungsverfahren und die Politik internationaler Organisationen. Bernhard Boockmann lehrt als außerplanmäßiger Professor an der Wirtschafts- und Sozialwissenschaftlichen Fakultät der Universität Tübingen, ist Mitglied im Ausschuss für Sozialpolitik des Vereins für Socialpolitik und Research Fellow des IZA-Netzwerkes.

Susanne Steffes absolvierte ein Studium der Volkswirtschaftslehre an der Universität Mannheim, welches sie 2005 als Diplom-Volkswirtin abschloss. Seit 2005 arbeitet sie als wissenschaftliche Mitarbeiterin am Zentrum für Europäische Wirtschaftsforschung GmbH (ZEW), Mannheim. Im Jahr 2010 schloss sie ihre Promotion im Fach Volkswirtschaftslehre bei Prof. Fitzenberger, Ph.D. an der Albert-LudwigsUniversität, Freiburg i. Br. zum Thema „Employment Dynamics and Heterogeneous Labor Markets: An Analysis with Linked EmployerEmployee and Company Data“ ab. Ihre aktuellen Forschungsfelder sind: empirische Arbeitsmarktforschung, insbesondere Beschäftigungsdynamik und Karriereverläufe sowie Personalökonomik, insbesondere der Einfluss von Human Resource Management auf die Beschäftigung in Unternehmen. 